\title{
Dermoscopy of Lupus Miliaris Disseminatus Faciei Lesions in Different Stages of Evolution
}

\author{
Pankhuri Dudani ${ }^{1}$, Nikhil Mehta $^{1}$
}

1 Department of Dermatology and Venereology, All India Institute of Medical Sciences, Delhi, India

Key words: dermatoscopy, lupus miliaris disseminatus faciei, telangiectasia, in-focus vessels, varioliform scar

Citation: Dudani P, Mehta N. Dermoscopy of lupus miliaris disseminatus faciei lesions in different stages of evolution. Dermatol Pract Concept. 2022;12(1):e2022017. DOI: https://doi.org/10.5826/dpc.1201a17

Accepted: May 26, 2021; Published: January 2022

Copyright: $@ 2022$ Dudani and Mehta. This is an open-access article distributed under the terms of the Creative Commons AttributionNonCommercial License (BY-NC-4.0), https://creativecommons.org/licenses/by-nc/4.0/, which permits unrestricted noncommercial use, distribution, and reproduction in any medium, provided the original authors and source are credited.

Funding: None.

Competing interests: None.

Authorship: Both authors have contributed significantly to this publication.

Corresponding author: Nikhil Mehta, MD, Department of Dermatology and Venereology.

All India Institute of Medical Sciences, Delhi, India. E-mail: nikhilmehtadermatology@gmail.com

\section{Introduction}

Lupus miliaris disseminatus faciei (LMDF) is an uncommon chronic granulomatous disease of the head and neck region. Its dermoscopic features have recently been described, mainly in Fitzpatrick skin-types 2 and 3. Its etiology is unknown, and adults between the second and fourth decades of life are affected most.

\section{Case Presentation}

A 35-year-old man, Fitzpatrick skin-type 4, presented with asymptomatic yellowish-to-red-colored papules over the forehead, eyelids, nose, and ear helices; and elongated plaques over the neck, for 5 months. Many lesions had healed with depressed varioliform and boxcar-like scars. Dermoscopy of dome-shaped active papules showed structureless yellow-red areas, follicular plugs, and in-focus telangiectasias (Figure 1, $\mathrm{A}$ and D). The latter were most prominent peripherally but were also seen centrally. Few lesions showed a central white stellate region. Early depressed scars showed in-focus marginal telangiectasias (Figure 1, B and E). Elongated plaques over neck showed similar features of yellow-red structureless areas and white areas suggestive of early scarring in a linear pattern, along with short linear and branching vessels arranged radially (Figure 1, C and F). Histopathology showed multiple perifollicular necrotizing epithelioid cell granulomas in the dermis consistent with LMDF (Figure 2, A and B).

\section{Conclusions}

LMDF presents as multiple skin-colored to erythematous fleshy papules and plaques over cheeks, periocular and perioral regions, especially over eyelids and earlobes. At neck, they present as elongated plaques. Lesions resolve spontaneously in 1-2 years or faster with treatment, leaving depressed, atrophic, varioliform scars. 


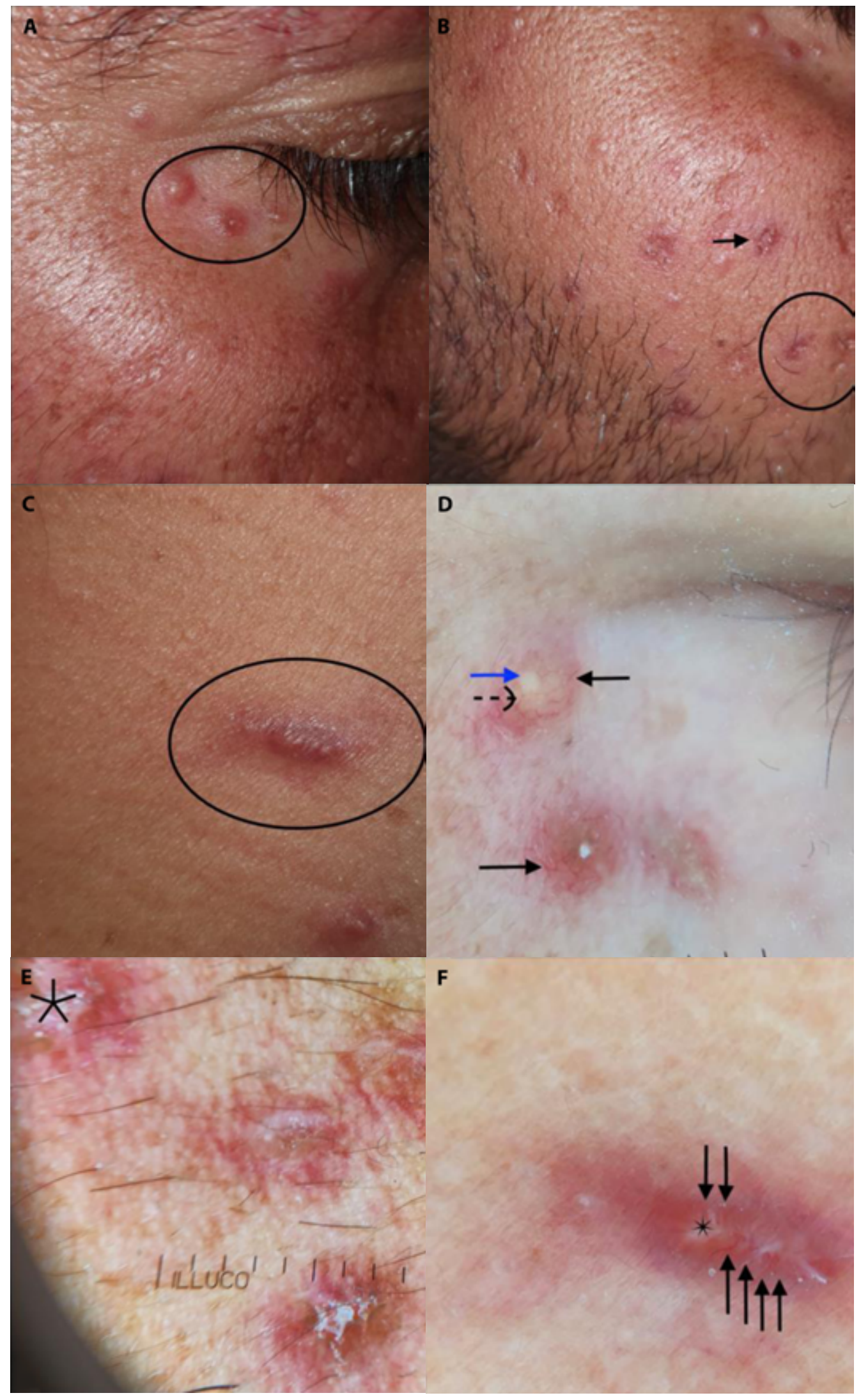

Figure 1. (A) Erythematous to skin-colored papules over upper and lower eyelid, with middle papule showing central depression. (B) Multiple depressed erythematous scars over the right cheek. (C) Linear erythematous elevated plaques over the right side of neck with background erythema. (D) Dermoscopy of lesions circled in A, showing follicular plug (blue arrow), peripheral in-focus telangiectasias extending to the central region (dotted arrow); white central follicular scar is also seen. (E) Telangiectasias are seen in all lesions corresponding to depressed scars in B, with a central white follicular scar. It is classically stellate, seen in lesion marked as an asterisk. (F) Linear yellowish region (asterisk) and multiple in-focus short linear and linear branching vessels along the lesion (black arrows). 
Histopathologically, LMDF is characterized by follicular plugs and a superficial dermal perifollicular granulomatous infiltrate with or without caseation. Late lesions show extensive perifollicular fibrosis.

Dermoscopic features include follicular plugs, some resembling a target surrounding the center, with short linear and linear-branching vessels on an orange-yellow/ erythematous background. Different stages of lesions have different dermoscopic features. With chronicity, there is an increase in follicular plugs and perifollicular scaling. The yellow perifollicular background is replaced by white structures in late lesions, corresponding to fibrosis replacing the granulomas [1].

Dermoscopic findings of LMDF differ from other papular granulomatous facial disorders, such as papular sarcoidosis, granulomatous rosacea, lupus vulgaris, and post-kala-azar dermal leishmaniasis (Table 1). Papular sarcoidosis shows orange-yellow structureless areas and does not show follicular plugs and stellate scars. Granulomatous rosacea shows vascular polygons not seen in LMDF.

Our case showed structureless yellow-orange regions, follicular plugs, and white structures as previously described [2]. In addition to documenting these features in elongated plaques, we were able to see in-focus telangiectasias, both in inflammatory lesions as well as in lesions evolving into depressed scars. Some of these vessels were reaching the center of the lesions. Earlier reports have noted unfocussed arborizing, comma-shaped and linear vessels. Yellow areas and vascular changes were readily seen in our patient despite a darker skin phenotype. However, these observations are from one case and may be extrapolated if noted in more patients.

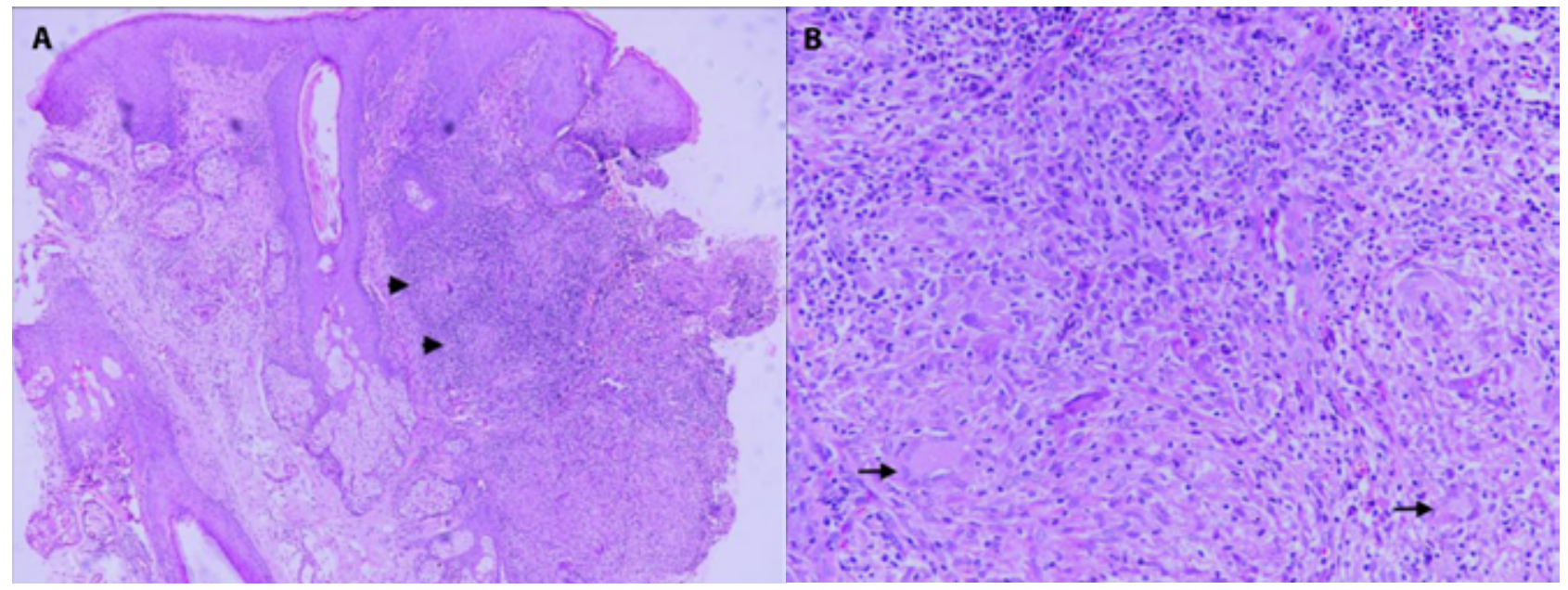

Figure 2. Histopathological examination of facial papule showing features of lupus miliaris disseminates faciei. (A) Irregular acanthosis of the epidermis and dermal perifollicular dense infiltrate with epithelioid cell granulomas (arrowhead) (H\&E, $\times 100)$. (B) Higher magnification; the infiltrate is composed of lymphocytes, epithelioid cells, and Langhans giant cells (arrow) (H\&E, $\times 400)$.

Table 1. Dermoscopy of Various Papular Granulomatous Disorders on the Face

\begin{tabular}{|l|l|}
\hline \multicolumn{1}{|c|}{ Disorder } & \multicolumn{1}{c|}{ Dermoscopic Findings } \\
\hline Sarcoidosis & $\begin{array}{l}\text { Orange-yellow structureless areas with well-focused linear } \\
\text { and branching vessels; white scar-like depigmented areas }\end{array}$ \\
\hline Granulomatous rosacea & $\begin{array}{l}\text { Linear reddish-violaceous vessels arranged in polygonal } \\
\text { network with diffuse or localized orange areas }\end{array}$ \\
\hline Lupus vulgaris & $\begin{array}{l}\text { Yellowish-white globules, milia-like cysts, white } \\
\text { structureless areas } \\
\text { Pinkish red background, with telangiectasias (linear, } \\
\text { branching) } \\
\text { White scales, shiny white streaks, white rosettes } \\
\text { A bluish hue may be seen }\end{array}$ \\
\hline Post-kala-azar dermal leishmaniasis & Multiple yellow tears and erythema \\
\hline Granuloma faciale & $\begin{array}{l}\text { Translucent white-gray background with whitish streaks } \\
\text { and linear telangiectasia }\end{array}$ \\
\hline
\end{tabular}


Dermoscopic features of LMDF lesions vary with time and can include in-focus telangiectasias in all stages of evolution, including early scars.

Informed consent: Written informed consent for publication of clinical details and clinical images was obtained from the patient.

\section{References}

1. Litaiem N, Chamli A, Bacha T, et al. Dermoscopic features of lupus miliaris disseminates faciei: Distinct aspects depending on disease stage. Clin Case Rep. 2020;8(9):1793-1796. DOI: 10.1002/ ccr3.2979. PMID: 32983497. PMCID: PMC7495779.

2. Chauhan P, Jindal R, Shirazi N. Dermoscopy of lupus miliaris disseminatus faciei: a step closer to diagnosis. Dermatol Pract Concept. 2020;10(3):e2020055. DOI: 10.5826/dpc.1003a55. PMID: 32685274; PMCID: PMC7346596. 